\title{
Potencial de veiculação dos ovos de Dermatobia hominis (Linnaeus Jr., 1781) por Cochliomyia macellaria (Fabricius, 1775), em laboratório*
}

\section{Carrying potential of Dermatobia hominis (Linnaeus Jr., 1781) eggs by Cochliomyia macellaria (fabricius, 1775), at laboratory}

\author{
Virginio Pereira da Silva Junior, ${ }^{* \star}$ André de Souza Leandro, ${ }^{* \star}$ Gonzalo Efrain Moya Borja ${ }^{\star \star}$
}

\begin{abstract}
Resumo
O comportamento de oviposição de Dermatobia hominis (Linnaeus Jr., 1781) (Diptera : Cuterebridae) sobre adultos de Cochliomyia macellaria (Fabricius, 1775) (Diptera : Calliphoridae), foi estudado em condições de laboratório no Instituto de Biologia da Universidade Federal Rural do Rio de Janeiro. Trinta fêmeas e 30 machos de $D$. hominis foram colocados em gaiolas, durante três dias, com 60 machos e 60 fêmeas de $C$. macellaria. Vetores carreando ovos de $D$. hominis foram coletados e separados por sexo. Os resultados indicaram que $12(20 \%)$ de 60 fêmeas de $C$. macellaria e seis $(10 \%)$ de 60 machos estavam carreando ovos de $D$. hominis. Não houve diferença significativa, como determinado pelo $\mathrm{X}^{2}$. Entretanto, fêmeas de $D$. hominis mostraram preferência para ovipositar em fêmeas de $C$. macellaria.
\end{abstract}

Palavras-chave: Dermatobia hominis, berne; vetor; Cochliomyia macellaria.

\section{Introdução}

Dermatobia hominis(Linnaeus Jr, 1981) (Diptera: Cuterebridae), a mosca do "Berne", é um díptero, cujas larvas são parasitas obrigatórios e têm como hospedeiro o homem e os animais domésticos, principalmente o gado bovino. Suas larvas desenvolvem o parasitismo no tecido cutâneo dos animais, determinando uma miíase primária do tipo nodular (Creighton \& Neel, 1952; Guimarães \& Papavero, 1966).

A D. hominis apresenta características de oviposição peculiares, na qual captura outro díptero com as patas em pleno vôo, fixando uma massa de ovos sobre a parte láteroventral do abdome (Guimarães \& Papavero, 1966). Como vetores dos ovos de $D$. hominis, alguns membros das famílias Culicidae, Simulidae, Tabanidae, Anthomyidae, Muscidae, Sarcophagidae, Cuterebridae e Tephiridae, foram listados, sendo o muscideo Sarcopromusca pruna considerado o mais importante dos vetores (Artigas \& Serra, 1965; Guimarães \& Papavero, 1966; Mourier \& Banegas, 1970). O potencial de veiculação dos ovos da $D$. hominis por S. pruna, em laboratório, foi de $37,03 \%$ para fêmeas e 22,23\% para machos (Pedroso, 1990).

A dermatobiose é uma parasitose que causa grandes perdas econômicas à pecuária, principalmente pela depreciação do couro e pela queda na produção de carne e leite (Andersen, 1962; Steelman, 1976; Lopes,1979).
Cochliomyia macellaria (Fabricius,"1775) (Diptera: Calliphoridae) é uma espécie que ocorre desde o Sul do Canadá até a Patagônia e Chile (Ferreira, 1983). As larvas dessa espécie são necrobiontófagas, sendo invasora secundária de ferimentos (Laake et al., 1936).

Um exemplar de $C$. macellaria foi capturado no Brasil portando ovos de $D$. hominis (Almeida, 1933). O califorídeo Lucilia cuprina (Wiedemann, 1830), comportou-se como vetor dos ovos de $D$. hominissob condições de laboratório (Lima et al., 1997).

O objetivo do presente estudo foi verificar o potencial de veiculação dos ovos de $D$. hominispela $C$. macellaria em laboratório e a influência do sexo na veiculação.

\section{Material e métodos}

O trabalho foi desenvolvido no laboratório de Entomologia do Instituto de Biologia da Universidade Federal Rural do Rio de Janeiro. Foi estabelecida uma colônia de Cochiomyia macellaria, em laboratório, a partir de moscas capturadas com rede entomológica, em áreas da Estação para Pesquisas Parasitológicas W. O. Neitz da UFRRJ. As moscas capturadas foram trazidas para o laboratório, acondicionadas em gaiolas teladas $(30 \times 30 \times 30 \mathrm{~cm})$ e alimentadas com solução de glicose a $20 \%$ e água. As gaiolas contendo os adultos selvagens foram mantidas em condições de

\footnotetext{
* Trabalho realizado com auxílio do CNPq.

** Curso de Pós-Graduação em Medicina Veterinária - Parasitologia Veterinária, Departamento de Parasitologia Animal, Instituto de Biologia, Universidade Federal Rural do Rio de Janeiro, Km 47 da Antiga Rodovia Rio-São Paulo, 23890-000, Seropédica, Rio de Janeiro, Brasil.
} 
laboratório (24 a $28^{\circ} \mathrm{C}$ e umidade relativa $70-80 \%$ ). Para o crescimento das larvas utilizou-se como meio de cultura, carne eqüina. No experimento foi utilizada a geração F1.

Os adultos de $D$. hominis foram obtidos através de coleta de larvas de $3^{\circ}$ instar (L3) da pele de bovinos recém-abatidos, em matadouros do Rio de Janeiro. As larvas foram extraídas, manualmente, e levadas para o laboratório, contadas e colocadas em recipientes contendo serragem, onde ficaram até a emergência dos adultos.

Utilizou-se seis gaiolas contendo cada uma, cinco casais de $D$. hominis, e 10 casais de $C$. macellaria. Ao longo de 72 horas foram coletados adultos de $C$. macellaria portando ovos de $D$. hominis.

\section{Resultados e discussão}

Na Tabela 1 encontram-se os resultados sobre a capacidade de veiculação dos ovos de $D$. hominis. Do total de 60 machos e 60 fêmeas, seis machos (10\%) portavam ovos de $D$. hominis, enquanto que 12 fêmeas $(20 \%)$ apresenta-

Tabela 1: Percentagem de Cochliomyia macellaria portando ovos de Dermatobia hominis, em laboratório

Sem ovos Com ovos Portando ovos

(\%)

\begin{tabular}{lrrr}
\hline Machos & 54 & 06 & 10 \\
Fêmeas & 48 & 12 & 20 \\
Total & 102 & 18 & 15 \\
\hline
\end{tabular}

ram-se portadoras. No total entre machos e fêmeas, 18 exemplares (15\%) portavam ovos. Não houve diferença, como determina o teste $X^{2}$ Entretanto, fêmeas de $D$. hominis mostraram preferência para ovipositar em fêmeas de C. macellaria. Pedroso (1990), relata que exemplares fêmeas de Sarcopromusca pruna são mais facilmente capturados pelas fêmeas de $D$. hominis, para postura.

A Figura 1 mostra exemplar de $C$. macellaria portando ovos de $D$. hominis. C. macellaria foi capturada portando ovos de $D$. hominis (Almeida,1933). Outro califorídeo capturado portando ovos no Rio Grande do Sul foi L. cuprina (Ribeiro et al., 1985). Esta espécie apresentou-se capaz de veicular ovos de $D$. hominis em laboratório, onde num total de 60 machos, $30,0 \%$ foram vetores e em 60 fêmeas, $26,7 \%$ portavam ovos (Lima et al., 1997).

No presente estudo observou-se postura dos ovos do berne em três exemplares de $D$. hominis.

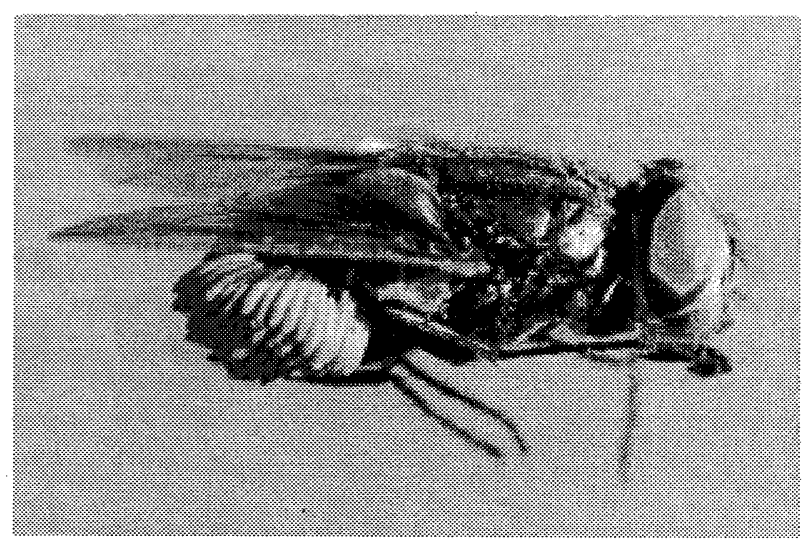

Figura 1: Cochliomyia macellaria portando ovos de Dermatobia hominis

\begin{abstract}
The oviposition behavior of Dermatobia hominis (Linnaeus, Jr., 1781) (Diptera: Cuterebridae) on adults Cochliomyia macellaria (Fabricius, 1775) (Diptera: Calliphoridae) was studied in laboratory conditions at the Institute of Biology of the UFRRJ. Thirty males and 30 females of $D$. hominis were caged during three days with 60 males and 60 females of $C$. macellaria. Vectors carrying eggs of $D$. hominis were collected and separated by sex. Results indicated that $12(20 \%)$ out of $60 \mathrm{C}$. macellaria females and six (10\%) out of $60 \mathrm{C}$. macellaria males were carrying eggs of $D$. hominis. There was no significant difference as determined by $\mathrm{X}^{2}$ test, however, $D$. hominis females showed some preference to oviposition females of C. macellaria.
\end{abstract}

Keywords: Dermatobia hominis; berne; vector; Cochliomyia macellaria.

\section{Referências bibliográficas}

ALMEIDA, J. L. Nouveaux agents de transmission de la berne Dermatobia hominis (L. junior, 1781) en Brésil. Societé de Biologie Paris, Compts Rendus, v. 113, n. 27, p. 1274-1275, 1993.

ANDERSEN, E. W. Control of the Dermatobia hominis in Central America. Vet. Rec., v. 74, n. 28, p. 784-786, 1962.
ARTIGAS , P. T. \& SERRA, R. G. Portadores de ovos de Dermatobia hominis (Linnaeus $\mathrm{Jr}, 1781$ ). Atualização da lista de foréticos, com enumeração de novos agentes transmissores do berne. Cien. Cult. (Brasil) v. 17, n. 1, p. 21-29, 1965.

CREIGHTON, J. T. \& NEEL, W. W., 1952. Biologia y combate del tórsalo o nuche, Dermatobia hominis (L. Jr.) . Reseña Bibliográfica, Turrialba v. 2, n. 2, p. 59-65. 
FERREIRA, M. J. M. Sinantropia de Calliphoridae (Diptera) em Goiânia, Goiás. Rev. Bras. Biol. v. 43, n. 2, p. 199-210, 1983.

GUIMARÃES, J. H. \& PAPAVERO, N. A tentative annotated bibliography of Dermatobia hominis (Linnaeus Jr. 1781) (Diptera: Cuterebridae). Arq. Zool., v. 14, n. 4, p. 223-294, 1966.

LAAKE, E. W.; CUSHING, E. C. \& PARISH, H. E. Biology of the primary screwworm fly, Cochliomyia americana, and a comparison of it's stages with those of $C$. macellaria. Washington, USDA. Tech. Bull. v. 500, p. 24, 1936.

LIMA, M. A. M. ; SILVA JUNIOR, V. P. \& BORJA, G. E. M. Lucilia cuprina (Wied., 1830) vetor dos ovos de Dermatobia hominis (Linnaeus Jr., 1781) no laboratório. Resumos do $16^{\circ}$ Congresso Brasileiro de Entomologia, Salvador, BA, p. 259, 1997.

LOPES, V. G. El nuche. Vida e control. Agric. Amer. v. 10, p. 34$36,1979$.
MOURIER, H. \& BANEGAS, A. D. Observations on the oviposition nad the ecology of the eggs of Dermatobia hominis (Diptera: Cuterebridae). Vidensk. Med. Dan. Naturhist. Foren. v. 33, p. 59-68, 1970.

PEDRoso, D. Aspectos da Bioecologia, Morfologia das fases jovens e controle da Sarcopromusca pruna (Shanon \& Del Ponte, 1926) (Diptera: Muscidae). Tese de Doutorado em Ciências em Medicina Veterinária Parasitologia Veterinária. Rio de Janeiro. UFRRJ, 130 p. 1990.

RIBEIRO, P. B.; OLIVEIRA, C. M. B.; COSTA, P. R. P. \& BRUM, J. G. W. Foréticos da Dermatobia hominis (L. Jr., 1781) (Diptera: Cuterebridae) no Rio Grande do Sul, brasil. Arq. Bras. Med. Vet. Zoot., v. 37, n. 5, p. 507-509, 1985.

STEELMAN, C. D. Effects of external and internal arthropods parasites on domestic livestock production. Ann. Ver. Entom. v. 21, p. 155-178, 1976.

\section{H HEXÁGONO}

Química e Equipamentos para Laboratórios

\section{Distribuidor Merck}

Rua Souza Barros, $\mathrm{n}^{\circ} 560$ - Engenho Novo 20961-150 - Rio de Janeiro, RJ
Tel.: (021) 501-4536

Fax: (021) 501-3723 